To submit to the International Journal of Computer Mathematics Vol. 00, No. 00, Month 20XX, 1-

\title{
A note on finite difference methods for nonlinear fractional differential equations with non-uniform meshes
}

\author{
Yanzhi Liu ${ }^{\mathrm{a}}$ and Jason Roberts ${ }^{\mathrm{b}}$ and Yubin Yan ${ }^{\mathrm{c} *}$ \\ a Department of Mathematics, Lvliang University, Lishi, 033000, P.R. China, \\ 115027431@qq.com; ${ }^{b}$ Department of Mathematics, University of Chester, Thornton Science \\ Park, Pool Lane, Ince, CH2 4NU, j.roberts@chester.ac.uk; ${ }^{c}$ Department of Mathematics, \\ University of Chester, Thornton Science Park, Pool Lane, Ince, CH2 $4 \mathrm{NU}$
}

(Received 00 Month 20XX; final version received 00 Month 20XX)

\begin{abstract}
We consider finite difference methods for solving nonlinear fractional differential equations in the Caputo fractional derivative sense with non-uniform meshes. Under the assumption that the Caputo derivative of the solution of the fractional differential equation is suitably smooth, Li et al. "Finite difference methods with non-uniform meshes for nonlinear fractional differential equations", Journal of Computational Physics, 316(2016), 614-631, obtained the error estimates of finite difference methods with non-uniform meshes. However the Caputo derivative of the solution of the fractional differential equation in general has a weak singularity near the initial time. In this paper, we obtain the error estimates of finite difference methods with non-uniform meshes when the Caputo fractional derivative of the solution of the fractional differential equation has lower smoothness. The convergence result shows clearly how the regularity of the Caputo fractional derivative of the solution affect the order of convergence of the finite difference methods. Numerical results are presented that confirm the sharpness of the error analysis.
\end{abstract}

Keywords: nonlinear fractional differential equation, predictor-corrector method, error estimates, non-uniform meshes, trapezoid formula.

2010 AMS Subject Classification: 65M12; 65M06; 65M70;35S10

\section{Introduction}

In this paper, we will consider finite difference methods for solving the following fractional nonlinear differential equation, with $\alpha>0$,

$$
{ }_{0}^{C} D_{t}^{\alpha} y(t)=f(t, y(t)), t>0, y^{(k)}(0)=y_{0}^{(k)}, k=0,1, \ldots,\lceil\alpha\rceil-1,
$$

where the $y_{0}^{(k)}$ may be arbitrary real numbers and ${ }_{0}^{C} D_{t}^{\alpha} y(t)$ denotes the Caputo fractional derivative defined by

$$
{ }_{0}^{C} D_{t}^{\alpha} y(t)=\frac{1}{\Gamma(\lceil\alpha\rceil-\alpha)} \int_{0}^{t}(t-s)^{\lceil\alpha\rceil-\alpha-1} y^{\lceil\alpha\rceil}(s) d s,
$$

where $\lceil\alpha\rceil$ is the smallest integer $\geq \alpha$. As usual we demand that the function $f$ is continuous and fulfills a Lipschitz condition with respect to its second argument with

\footnotetext{
*Corresponding author. Email: y.yan@chester.ac.uk
} 
Lipschitz constant $L$ on a suitable set $G$. Under these assumptions, Diethelm et al. [? , Theorems 2.1,2.2] showed that (??) has a unique solution $y$ on some interval $[0, T]$.

It is well-known that (??) is equivalent to, [? , Lemma 2.3]

$$
y(t)=\sum_{\nu=0}^{\lceil\alpha\rceil-1} y_{0}^{(\nu)} \frac{t^{\nu}}{\nu !}+\frac{1}{\Gamma(\alpha)} \int_{0}^{t}(t-s)^{\alpha-1} f(s, y(s)) d s .
$$

Equations of this type arise in a number of applications where models based on fractional calculus are used, such as viscoelastic materials, anomalous diffusion, signal processing and control theory, etc., see Oldham and Spanier [? ], Kilbas et al. [?], Podlubny $[?]$.

It is not possible to find the analytic solution of (??) for the general $f$. Therefore we have to apply some numerical methods for solving (??). Stability and convergence of such numerical methods are analyzed under certain smoothness assumptions about the solutions or data for (??), see, for example, [?], [?], [?], [?], [?], [?], [?], [?], [?], [?].

Most analysis of the numerical methods for solving (??) is deduced under the assumptions that the meshes are uniform, see, for example, [?], [? ], [? ], [? ], [? ], [? ]. To obtain a higher order numerical method with uniform meshes, the solutions or data of (??) are required to be suitably smooth, for example, ${ }_{0}^{C} D_{t}^{\alpha} y \in C^{m}[0, T], m \geq 2$ in [? , Theorem 3.2]. However, as stated in [? , Theorem 2.2], although $y \in C^{m}[0, T]$ for some $m \in \mathbb{N}, 0<\alpha<m$, the Caputo fractional derivative ${ }_{0}^{C} D_{t}^{\alpha} y$ behaves as $t^{\lceil\alpha\rceil-\alpha}$ when $y^{\lceil\alpha\rceil}(0) \neq 0, \alpha>0$. Therefore it is interesting to design some numerical methods which have the optimal convergence orders when ${ }_{0}^{C} D_{t}^{\alpha} y$ has the lower smoothness. One way is to use the numerical methods with non-uniform meshes. In this paper, we will assume that ${ }_{0}^{C} D_{t}^{\alpha} y$ satisfies the following smooth assumption. For simplicity of the notations, we only consider the case with $0<\alpha<2$. Similarly one may consider the case with $\alpha \geq 2$.

Assumption 1 Let $0<\sigma<1$ and let $g(t):={ }_{0}^{C} D_{t}^{\alpha} y(t)$ with $0<\alpha<2$. There exists a constant $c>0$ such that

$$
\left|g^{\prime}(t)\right| \leq c t^{\sigma-1}, \quad\left|g^{\prime \prime}(t)\right| \leq c t^{\sigma-2} .
$$

Here $g^{\prime}(t), g^{\prime \prime}(t)$ denote the first and second derivatives of $g$, respectively.

It is obvious that the smoothness of $g$ in Assumption ?? is weaker than $g \in C^{2}[0, T]$. In other words, the Assumption ?? has less requirements for the smoothness of $g$ than $g \in C^{2}[0, T]$. Further we note that the Assumption ?? is reasonable in the application. Below we show some cases where $g(t):={ }_{0}^{C} D_{t}^{\alpha} y(t)$ satisfies the Assumption ??.

- By [? , Lubich, 1983, Theorem 2.1], assume that $f \in C^{m}(G), m \geq 2$ and $\alpha \in(0,1)$, then, with some constants $c_{1}, c_{2}, \ldots, c_{\hat{\nu}} \in \mathbb{R}$,

$$
y=c_{1} t^{\alpha}+c_{2} t^{2 \alpha}+\cdots+c_{\hat{\nu}} t^{\hat{\nu} \alpha}+\text { smoother terms }
$$

which implies that, with some constants $d_{1}, d_{2}, \ldots, d_{\hat{\nu}} \in \mathbb{R}$,

$$
\begin{aligned}
g: & ={ }_{0}^{C} D_{t}^{\alpha} y=d_{1} t^{\alpha-\alpha}+d_{2} t^{2 \alpha-\alpha}+\cdots+d_{\hat{\nu}} t^{\hat{\nu} \alpha-\alpha}+\text { smoother terms } \\
& =d_{1}+d_{2} t^{\alpha}+\cdots+d_{\hat{\nu}} t^{(\hat{\nu}-1) \alpha}+\text { smoother terms. }
\end{aligned}
$$


Thus we see $g:={ }_{0}^{C} D_{t}^{\alpha} y$ behaves as $c+c t^{\alpha}$ which implies that $\left|g^{\prime}(t)\right| \leq c t^{\alpha-1}$ and $\left|g^{\prime \prime}(t)\right| \leq c t^{\alpha-2}$. Hence we may apply Assumption ?? with $\sigma=\alpha$ in this case.

- By [?, Theorem 2.2], although the solution $y$ is very smooth, the Caputo derivative of the solution ${ }_{0}^{C} D_{t}^{\alpha} y$ may not be smooth. For example $y(t)=t \in C^{\infty}[0, T]$, but ${ }_{0}^{C} D_{t}^{\alpha} y=c t^{1-\alpha} \notin C^{2}[0, T]$. We may apply the Assumption ?? with $\sigma=1-\alpha$ in this case.

- It is very common that both $y$ and the Caputo derivative ${ }_{0}^{C} D_{t}^{\alpha} y$ have lower smoothness in the fractional differential equation. For example, the fractional differential equation, with $0<\alpha<1$,

$$
{ }_{0}^{C} D_{t}^{\alpha} y(t)+y(t)=0, \quad t \in(0, T], \quad y(0)=1,
$$

has the exact solution $y(t)=E_{\alpha, 1}\left(-t^{\alpha}\right)$, where $E_{\alpha, \gamma}(z)$ is the Mittag-Leffler function defined by $E_{\alpha, \gamma}(z)=\sum_{k=0}^{\infty} \frac{z^{k}}{\Gamma(\alpha k+\gamma)}, \alpha, \gamma>0$. Hence we have

$$
{ }_{0}^{C} D_{t}^{\alpha} y(t)=-E_{\alpha, 1}\left(-t^{\alpha}\right)=-1-\frac{\left(-t^{\alpha}\right)}{\Gamma(\alpha+1)}-\frac{\left(-t^{\alpha}\right)^{2}}{\Gamma(2 \alpha+1)}-\ldots
$$

which implies that the regularity of ${ }_{0}^{C} D_{t}^{\alpha} y(t)$ behaves as $c+c t^{\alpha}, 0<\alpha<1$. Thus we may apply the Assumption ?? with $\sigma=\alpha$ in this case.

Recently Li et al. [?] considered the error estimates of the rectangle formula, trapezoid formula and the predictor-corrector scheme for solving (??) with non-uniform meshes when the solution is suitably smooth, see also [?], [?], [?], [?]. Under the Assumption ??, we shall consider in this paper the error estimates of the finite difference methods introduced in Li et al. [?] with the non-uniform meshes. We show that the optimal convergence orders of the numerical methods may be recovered when the Caputo derivative of the solution of the fractional differential equation has lower smoothness. Our analysis is based on the excellent work in Stynes et al. [? ], [? ], where the authors applied the graded meshes to recover the convergence order of the finite difference method for solving time-fractional diffusion equation when the solution is not sufficiently smooth.

The paper is organized as follows. In Section 2 we introduce three numerical schemes for solving (??) with non-uniform meshes, i.e., the rectangle formula, the trapezoid formula and the predictor-corrector scheme. In section 3, we prove the error estimates of these three numerical methods under the Assumption ??. Finally in Section 4, we give some numerical examples which show that the numerical results are consistent with the theoretical results.

Throughout, the notations $C$ and $c$, with or without a subscript, denote generic constants, which may differ at different occurrences, but are always independent of the mesh size.

\section{Numerical methods}

In this section, we will introduce the different numerical methods for solving (??), see Li et al. [?], Diethelm [?].

Let $N \geq 1$ be a positive integer and let $0=t_{0}<t_{1}<\cdots<t_{N}=T$ be the non-uniform meshes on $[0, T]$. For simplicity, we assume that $T=1$. Let $\mu=\frac{2 T}{N(N+1)}$. Assume that 
the non-uniform meshes satisfy, [?]

$$
\tau_{j}=t_{j+1}-t_{j}=(j+1) \mu, j=0,1,2, \ldots, N-1 .
$$

Let us consider the following integral, with $n=0,1,2, \ldots, N-1$,

$$
I_{n+1}=\int_{0}^{t_{n+1}}\left(t_{n+1}-s\right)^{\alpha-1} f(s, y(s)) d s=\sum_{j=0}^{n} \int_{t_{j}}^{t_{j+1}}\left(t_{n+1}-s\right)^{\alpha-1} f(s, y(s)) d s .
$$

It can be approximated by the following approach

$$
I_{n+1} \approx \sum_{j=0}^{n} \int_{t_{j}}^{t_{j+1}}\left(t_{n+1}-s\right)^{\alpha-1} \tilde{f}_{j}(s, y(s)) d s
$$

where $\tilde{f}_{j}(s, y(s)), j=0,1,2, \ldots, n$ is the approximation of $f(s, y(s))$ on the interval $\left[t_{j}, t_{j+1}\right]$.

It will lead to different scheme by choosing different $\tilde{f}_{j}(s, y(s))$. Here we choose three kinds of $\tilde{f}_{j}(s, y(s))$ to derive the fractional rectangle, trapezoid, and predictor-corrector methods respectively, see $\mathrm{Li}$ et al. [? ]. Let $y_{n} \approx y\left(t_{n}\right), n=0,1,2, \ldots, N$ denote the approximate solution of $y\left(t_{n}\right)$.

(i). By choosing $\tilde{f}_{j}(s, y(s))$ as

$$
\tilde{f}_{j}(s, y(s))=f\left(t_{j}, y\left(t_{j}\right)\right) \text {, on }\left[t_{j}, t_{j+1}\right], j=0,1,2, \ldots, n,
$$

the fractional rectangle method for solving (??) is derived as, with $0<\alpha<2$,

$$
y_{n+1}=y(0)+y^{\prime}(0) t_{n+1}+\sum_{j=0}^{n} w_{j, n+1} f\left(t_{j}, y_{j}\right)
$$

where, with $j=0,1,2, \ldots, n$,

$$
w_{j, n+1}=\frac{1}{\Gamma(\alpha)} \int_{t_{j}}^{t_{j+1}}\left(t_{n+1}-s\right)^{\alpha-1} d s=\frac{\left(t_{n+1}-t_{j}\right)^{\alpha}-\left(t_{n+1}-t_{j+1}\right)^{\alpha}}{\Gamma(\alpha+1)} .
$$

We remark that when $0<\alpha<1$, there is no term $y^{\prime}(0) t_{n+1}$ at the right hand of (??). We will not repeat this remark below for $0<\alpha<1$.

(ii). If $\tilde{f}_{j}(s, y(s))$ is selected as, with $j=0,1,2, \ldots, n$,

$$
\tilde{f}_{j}(s, y(s))=\frac{s-t_{j+1}}{t_{j}-t_{j+1}} f\left(t_{j}, y\left(t_{j}\right)\right)+\frac{s-t_{j}}{t_{j+1}-t_{j}} f\left(t_{j+1}, y\left(t_{j+1}\right)\right), \text { on }\left[t_{j}, t_{j+1}\right],
$$

the fractional trapezoid method is derived as, with $0<\alpha<2$,

$$
y_{n+1}=y(0)+y^{\prime}(0) t_{n+1}+\sum_{j=0}^{n+1} \tilde{w}_{j, n+1} f\left(t_{j}, y_{j}\right)
$$


where

$$
\tilde{w}_{j, n+1}=\frac{1}{\Gamma(\alpha+2)}\left\{\begin{array}{cc}
\frac{1}{t_{1}} A_{0}, & \text { if } j=0, \\
\frac{1}{t_{j+1}-t_{j}} A_{j}+\frac{1}{t_{j-1}-t_{j}} B_{j}, & \text { if } j=1,2, \ldots, n, \\
\left(t_{n+1}-t_{n}\right)^{\alpha}, & \text { if } j=n+1,
\end{array}\right.
$$

and

$$
\begin{aligned}
& A_{0}=\left(t_{n+1}-t_{1}\right)^{\alpha+1}-t_{n+1}^{\alpha+1}+(\alpha+1) t_{1} t_{n+1}^{\alpha} \\
& A_{j}=\left(t_{n+1}-t_{j+1}\right)^{\alpha+1}-\left(t_{n+1}-t_{j}\right)^{\alpha+1}+(\alpha+1)\left(t_{j+1}-t_{j}\right)\left(t_{n+1}-t_{j}\right)^{\alpha}, \\
& B_{j}=\left(t_{n+1}-t_{j}\right)^{\alpha+1}-\left(t_{n+1}-t_{j-1}\right)^{\alpha+1}+(\alpha+1)\left(t_{j}-t_{j-1}\right)\left(t_{n+1}-t_{j}\right)^{\alpha} .
\end{aligned}
$$

Note that (??) is an implicit scheme. In order to decrease computational complexity, it is natural to introduce the following predictor-corrector method.

(iii) The predictor-corrector method

$$
\begin{aligned}
& y_{n+1}^{P}=y(0)+y^{\prime}(0) t_{n+1}+\sum_{j=0}^{n} w_{j, n+1} f\left(t_{j}, y_{j}\right), \\
& y_{n+1}=y(0)+y^{\prime}(0) t_{n+1}+\left(\sum_{j=0}^{n} \tilde{w}_{j, n+1} f\left(t_{j}, y_{j}\right)+\tilde{w}_{n+1, n+1} f\left(t_{n+1}, y_{n+1}^{P}\right)\right),
\end{aligned}
$$

where $w_{j, n+1}$ and $\tilde{w}_{j, n+1}$ are defined in (??) and (??), respectively.

\section{Error estimates}

In this section, under the Assumption ?? we shall consider the error estimates for the rectangle formula, trapezoid formula and predictor-corrector scheme introduced in Section 2 with non-uniform meshes.

\subsection{Rectangle formula}

In this subsection we will consider the error estimates of the rectangle formula (??) for solving (??). Assume that ${ }_{0}^{C} D_{t}^{\alpha} y \in C^{1}[0, T], \alpha>0$, Li et al. [?] proved the following theorem

Theorem 3.1 ([? , Theorem 4.1]) Let $\alpha>0$. If ${ }_{0}^{C} D_{t}^{\alpha} y \in C^{1}[0, T]$ and the nonequidistant stepsize is non-decreasing, then the rectangle formula (??) for equation (??) has the following error estimates

$$
\max _{0 \leq j \leq N}\left|y_{j}-y\left(t_{j}\right)\right| \leq C \tau_{\max }
$$

where $\tau_{\max }$ denotes the maximum of the step sizes, i.e.,

$$
\tau_{\max }=\max _{0 \leq j \leq N-1}\left(t_{j+1}-t_{j}\right)
$$


We will show the following result:

TheOREM 3.2 Let $0<\alpha<2$ and assume that $g:={ }_{0}^{C} D_{t}^{\alpha} y$ satisfies Assumption ??. Let $\tau_{j}, j=0,1,2, \ldots, N-1$ be the non-uniform meshes defined in (??). Assume that $y\left(t_{j}\right)$ and $y_{j}$ are the solutions of (??) and (??), respectively.

(1) If $0<\alpha \leq 1$, then we have

$$
\max _{0 \leq j \leq N}\left|y\left(t_{j}\right)-y_{j}\right| \leq\left\{\begin{array}{cc}
C N^{-2(\alpha+\sigma)}, & \text { if } 0<2(\alpha+\sigma)<1 \\
C N^{-2(\alpha+\sigma)} \ln (N), & \text { if } 2(\alpha+\sigma)=1 \\
C N^{-1}, & \text { if } 2(\alpha+\sigma)>1
\end{array}\right.
$$

(2) If $1<\alpha<2$, then we have

$$
\max _{0 \leq j \leq N}\left|y\left(t_{j}\right)-y_{j}\right| \leq C N^{-1}
$$

To prove Theorem ??, we need some preliminary lemmas.

Lemma 3.3 Let $0<\alpha<2$ and assume that $g:={ }_{0}^{C} D_{t}^{\alpha} y$ satisfies Assumption ??. Let $\tau_{j}, j=0,1,2, \ldots, N-1$ be the non-uniform meshes defined in (??).

- If $0<\alpha \leq 1$, then we have, with $n=0,1,2, \ldots, N-1, N \geq 1$,

$$
\left|\frac{1}{\Gamma(\alpha)} \sum_{j=0}^{n} \int_{t_{j}}^{t_{j+1}}\left(t_{n+1}-s\right)^{\alpha-1} g(s) d s-\sum_{j=0}^{n} w_{j, n+1} g\left(t_{j}\right)\right| \leq\left\{\begin{array}{cl}
C N^{-2(\alpha+\sigma)}, & \text { if } 0<2(\alpha+\sigma)<1, \\
C N^{-2(\alpha+\sigma)} \ln (N), & \text { if } 2(\alpha+\sigma)=1, \\
C N^{-1}, & \text { if } 2(\alpha+\sigma)>1 .
\end{array}\right.
$$

- If $1<\alpha<2$, then we have, with $n=0,1,2, \ldots, N-1, N \geq 1$,

$$
\left|\frac{1}{\Gamma(\alpha)} \sum_{j=0}^{n} \int_{t_{j}}^{t_{j+1}}\left(t_{n+1}-s\right)^{\alpha-1} g(s) d s-\sum_{j=0}^{n} w_{j, n+1} g\left(t_{j}\right)\right| \leq C N^{-1}
$$

Proof. Note that, with $n=2,3, \ldots, N-1, N \geq 3$, (it is easy to consider the cases for $N=1,2$, we omit these cases here)

$$
\begin{aligned}
& \left|\frac{1}{\Gamma(\alpha)} \sum_{j=0}^{n} \int_{t_{j}}^{t_{j+1}}\left(t_{n+1}-s\right)^{\alpha-1} g(s) d s-\sum_{j=0}^{n} w_{j, n+1} g\left(t_{j}\right)\right| \\
& =\frac{1}{\Gamma(\alpha)}\left|\sum_{j=0}^{n} \int_{t_{j}}^{t_{j+1}}\left(t_{n+1}-s\right)^{\alpha-1}\left(g(s)-g\left(t_{j}\right)\right) d s\right| \\
& \leq \frac{1}{\Gamma(\alpha)}\left|\int_{0}^{t_{1}}\left(t_{n+1}-s\right)^{\alpha-1}\left(g(s)-g\left(t_{0}\right)\right) d s\right|+\frac{1}{\Gamma(\alpha)}\left|\sum_{j=1}^{n-1} \int_{t_{j}}^{t_{j+1}}\left(t_{n+1}-s\right)^{\alpha-1}\left(g(s)-g\left(t_{j}\right)\right) d s\right| \\
& \quad+\frac{1}{\Gamma(\alpha)}\left|\int_{t_{n}}^{t_{n+1}}\left(t_{n+1}-s\right)^{\alpha-1}\left(g(s)-g\left(t_{n}\right)\right) d s\right| \\
& =I_{1}+I_{2}+I_{3} .
\end{aligned}
$$


For $I_{1}$, we have, by Assumption ??,

$$
\begin{aligned}
I_{1} & \leq C\left|\int_{0}^{t_{1}}\left(t_{n+1}-s\right)^{\alpha-1} \int_{0}^{s} g^{\prime}(\tau) d \tau d s\right| \leq C \int_{0}^{t_{1}}\left(t_{n+1}-s\right)^{\alpha-1} \int_{0}^{s} \tau^{\sigma-1} d \tau d s \\
& \leq C \int_{0}^{t_{1}}\left(t_{n+1}-s\right)^{\alpha-1} s^{\sigma} d s .
\end{aligned}
$$

If $0<\alpha \leq 1$, then we have, by Assumption ?? and (??),

$$
\begin{aligned}
I_{1} & \leq C\left(t_{n+1}-t_{1}\right)^{\alpha-1} \int_{0}^{t_{1}} s^{\sigma} d s \leq C\left(t_{n+1}-t_{1}\right)^{\alpha-1}\left(t_{1}\right)^{\sigma+1} \\
& =C\left(\frac{n(n+3)}{N(N+1)}\right)^{\alpha-1}\left(\frac{2}{N(N+1)}\right)^{\sigma+1} \leq C N^{-2(\alpha-1)} n^{2(\alpha-1)} N^{-2(\sigma+1)} \leq C N^{-2(\alpha+\sigma)} .
\end{aligned}
$$

If $1<\alpha<2$, then we have,

$$
\begin{aligned}
I_{1} & \leq C t_{n+1}^{\alpha-1} t_{1}^{\sigma+1}=C\left(\frac{(n+1)(n+2)}{N(N+1)}\right)^{\alpha-1}\left(\frac{2}{N(N+1)}\right)^{\sigma+1} \\
& \leq C n^{2(\alpha-1)} N^{-2(\alpha-1)} N^{-2(\sigma+1)} \leq C(n / N)^{2(\alpha-1)} N^{-2 \sigma-2} \leq C N^{-2 \sigma-2} \leq C N^{-1}
\end{aligned}
$$

For $I_{2}$, we have, with $\xi_{j} \in\left(t_{j}, t_{j+1}\right), j=1,2, \ldots, n-1$ and $n=2,3, \ldots, N-1$,

$$
I_{2}=\frac{1}{\Gamma(\alpha)}\left|\sum_{j=1}^{n-1} \int_{t_{j}}^{t_{j+1}}\left(t_{n+1}-s\right)^{\alpha-1} g^{\prime}\left(\xi_{j}\right)\left(s-t_{j}\right) d s\right|
$$

By Assumption ??, we have, see Stynes et al. [?], with $n \geq 4$, i.e., $N \geq 5$, ( the case for $n<4$ is easy to consider and we omit this case below),

$$
\begin{aligned}
I_{2} \leq & C\left|\sum_{j=1}^{n-1}\left(t_{j+1}-t_{j}\right) t_{j}^{\sigma-1} \int_{t_{j}}^{t_{j+1}}\left(t_{n+1}-s\right)^{\alpha-1} d s\right| \\
\leq & C\left|\sum_{j=1}^{\left\lceil\frac{n-1}{2}\right\rceil-1}\left(t_{j+1}-t_{j}\right) t_{j}^{\sigma-1} \int_{t_{j}}^{t_{j+1}}\left(t_{n+1}-s\right)^{\alpha-1} d s\right| \\
& +C\left|\sum_{j=\left\lceil\frac{n-1}{2}\right\rceil}^{n-1}\left(t_{j+1}-t_{j}\right) t_{j}^{\sigma-1} \int_{t_{j}}^{t_{j+1}}\left(t_{n+1}-s\right)^{\alpha-1} d s\right| \\
= & I_{21}+I_{22},
\end{aligned}
$$

where $\left\lceil\frac{n-1}{2}\right\rceil$ is the smallest integer $\geq \frac{n-1}{2}$ with $n=4,5, \ldots, N-1, N \geq 5$.

We first consider the estimates for $I_{21}$. We have, with $n \geq 4$,

$$
I_{21} \leq C \sum_{j=1}^{\left\lceil\frac{n-1}{2}\right\rceil-1}\left(t_{j+1}-t_{j}\right)^{2} t_{j}^{\sigma-1}\left(t_{n+1}-t_{j+1}\right)^{\alpha-1}
$$


Note that

$$
\begin{aligned}
& t_{j}^{\sigma-1}=\left(\frac{j(j+1)}{N(N+1)}\right)^{\sigma-1} \leq C(j / N)^{2(\sigma-1)}, \\
& t_{j+1}-t_{j}=\frac{2(j+1)}{N(N+1)} \leq C j N^{-2}
\end{aligned}
$$

and, since $1 \leq j \leq\left\lceil\frac{n-1}{2}\right\rceil-1, n \geq 4$,

$$
\begin{aligned}
\left(t_{n+1}-t_{j+1}\right)^{\alpha-1} & =\left(\frac{(n-j)(n+j+3)}{N(N+1)}\right)^{\alpha-1} \\
& \leq C(n / 2)^{\alpha-1} n^{\alpha-1} N^{-2(\alpha-1)} \leq C(n / N)^{2(\alpha-1)}
\end{aligned}
$$

Thus, by (??), (??) and (??), we have, with $n \geq 4$,

$$
I_{21} \leq C \sum_{j=1}^{\left\lceil\frac{n-1}{2}\right\rceil-1}\left(j N^{-2}\right)^{2}(j / N)^{2(\sigma-1)}(n / N)^{2(\alpha-1)} .
$$

If $0<\alpha \leq 1$, we have, by (??) and noting that $(j / n)^{2(1-\alpha)} \leq C$ in this case,

$$
\begin{aligned}
I_{21} & \leq C \sum_{j=1}^{\left\lceil\frac{n-1}{2}\right\rceil-1} j^{2(\alpha+\sigma)-2} N^{-2(\sigma+\alpha)}(j / n)^{2(1-\alpha)} \leq C N^{-2(\sigma+\alpha)} \sum_{j=1}^{\left\lceil\frac{n-1}{2}\right\rceil-1} j^{2(\alpha+\sigma)-2} \\
& \leq\left\{\begin{array}{cc}
C N^{-2(\alpha+\sigma)}, & \text { if } 0<2(\alpha+\sigma)<1, \\
C N^{-2(\alpha+\sigma)} \ln (N), & \text { if } 2(\alpha+\sigma)=1, \\
C N^{-1}, & \text { if } 2(\alpha+\sigma)>1,
\end{array}\right.
\end{aligned}
$$

where the last inequality for $2(\alpha+\sigma)>1$ follows from

$$
C N^{-2(\sigma+\alpha)} \sum_{j=1}^{\left\lceil\frac{n-1}{2}\right\rceil-1} j^{2(\alpha+\sigma)-2} \leq C N^{-2(\sigma+\alpha)} \int_{0}^{N} x^{2(\alpha+\sigma)-2} d x \leq C N^{-1}
$$

If $1<\alpha<2$, then we have, by (??) and noting that $(n / N)^{2(\alpha-1)} \leq C$ in this case,

$$
I_{21} \leq C N^{-2 \sigma-2} \sum_{j=1}^{\left[\frac{n-1}{2}\right]-1} j^{2 \sigma} \leq C N^{-2 \sigma-2} \int_{0}^{N} x^{2 \sigma} d x \leq C N^{-1}
$$

For $I_{22}$, noting that, with $\left\lceil\frac{n-1}{2}\right\rceil \leq j \leq n-1, n \geq 4$,

$$
t_{j}^{\sigma-1}=\left(\frac{j(j+1)}{N(N+1)}\right)^{\sigma-1} \leq C(j / N)^{2(\sigma-1)} \leq C(n / N)^{2(\sigma-1)},
$$

and

$$
t_{j+1}-t_{j}=\frac{2(j+1)}{N(N+1)} \leq C j N^{-2} \leq C n N^{-2}
$$


we have

$$
\begin{aligned}
I_{22} & \leq C\left|\sum_{j=\left\lceil\frac{n-1}{2}\right\rceil}^{n-1}\left(n N^{-2}\right)(n / N)^{2(\sigma-1)} \int_{t_{j}}^{t_{j+1}}\left(t_{n+1}-s\right)^{\alpha-1} d s\right| \\
& \leq C n^{2 \sigma-1} N^{-2 \sigma} \int_{t_{\left\lceil\frac{n-1}{2}\right\rceil}}^{t_{n}}\left(t_{n+1}-s\right)^{\alpha-1} d s .
\end{aligned}
$$

Further we note that

$$
\begin{aligned}
& \int_{t_{\left\lceil\frac{n-1}{2}\right\rceil}}^{t_{n}}\left(t_{n+1}-s\right)^{\alpha-1} d s=\frac{1}{\alpha}\left[\left(t_{n+1}-t_{\left\lceil\frac{n-1}{2}\right\rceil}\right)^{\alpha}-\left(t_{n+1}-t_{n}\right)^{\alpha}\right] \\
& \leq \frac{1}{\alpha}\left(t_{n+1}-t_{\left\lceil\frac{n-1}{2}\right\rceil}\right)^{\alpha} \leq \frac{1}{\alpha} t_{n+1}^{\alpha}=\frac{1}{\alpha}((n+1)(n+2) / N(N+1))^{\alpha} \leq C(n / N)^{2 \alpha} .
\end{aligned}
$$

Thus we have, by (??), (??) and (??), with $\left\lceil\frac{n-1}{2}\right\rceil \leq j \leq n-1, n \geq 4$,

$$
I_{22}=C n^{2 \sigma-1} N^{-2 \sigma}(n / N)^{2 \alpha}=C N^{-2(\sigma+\alpha)} n^{2(\sigma+\alpha)-1} .
$$

If $0<\alpha \leq 1$, then we have

$$
I_{22} \leq\left\{\begin{array}{l}
C N^{-2(\sigma+\alpha)}, \quad \text { if } 0<2(\sigma+\alpha) \leq 1, \\
C N^{-1}, \quad \text { if } 2(\sigma+\alpha)>1,
\end{array}\right.
$$

where the last inequality follows from, with $2(\sigma+\alpha)>1$,

$$
I_{22} \leq C N^{-2(\sigma+\alpha)}(n / N)^{2(\sigma+\alpha)-1} N^{2(\sigma+\alpha)-1} \leq C N^{-1} .
$$

If $1<\alpha<2$, then we have, by (??), since $\alpha \in(1,2)$ implies $2(\sigma+\alpha)>1$,

$$
I_{22} \leq C N^{-1} \text {. }
$$

For $I_{3}$, we have, with $\xi_{n} \in\left(t_{n}, t_{n+1}\right), n=1,2, \ldots, N-1$,

$$
I_{3} \leq C\left|\int_{t_{n}}^{t_{n+1}}\left(t_{n+1}-s\right)^{\alpha-1} g^{\prime}\left(\xi_{n}\right)\left(s-t_{n}\right) d s\right| .
$$

By Assumption ??, we have, with $0<\alpha<2$

$$
\begin{aligned}
I_{3} & \leq C\left(t_{n+1}-t_{n}\right) t_{n}^{\sigma-1} \int_{t_{n}}^{t_{n+1}}\left(t_{n+1}-s\right)^{\alpha-1} d s \\
& =C\left(t_{n+1}-t_{n}\right) t_{n}^{\sigma-1} \frac{1}{\alpha}\left(t_{n+1}-t_{n}\right)^{\alpha} \leq C\left(t_{n+1}-t_{n}\right)^{1+\alpha} t_{n}^{\sigma-1} \\
& \leq C\left(n N^{-2}\right)^{1+\alpha}(n / N)^{2(\sigma-1)}=C n^{\alpha+2 \sigma-1} N^{-2(\alpha+\sigma)} \\
& \leq\left\{\begin{array}{cc}
C N^{-2(\sigma+\alpha)}, & \text { if } 2(\alpha+\sigma) \leq 1+\alpha, \\
C N^{-(1+\alpha)}, & \text { if } 2(\alpha+\sigma)>1+\alpha,
\end{array}\right.
\end{aligned}
$$


where the last inequality follows from, with $2(\alpha+\sigma) \geq 1+\alpha$,

$$
C n^{\alpha+2 \sigma-1} N^{-2(\alpha+\sigma)} \leq C(n / N)^{\alpha+2 \sigma-1} N^{\alpha+2 \sigma-1} N^{-2(\alpha+\sigma)} \leq C N^{-(1+\alpha)} .
$$

Obviously the bound for $I_{3}$ is stronger than the bound for $I_{21}$.

Together these estimates complete the proof of Lemma ??.

We also need the following lemmas.

Lemma 3.4 ([? , Lemma 3.1]) If $\alpha>0, n$ is a nonnegative integer, $\tau_{j} \leq \tau_{j+1} j=$ $0,1, \ldots, n-1$, then $w_{j, n+1}$ and $\tilde{w}_{j, n+1}$ defined by (??) and (??) respectively have the following estimates

$$
w_{j, n+1} \leq C_{\alpha} \tau_{j}\left(t_{n+1}-t_{j}\right)^{\alpha-1}, \quad j=0,1, \ldots, n,
$$

and

$$
\tilde{w}_{j, n+1} \leq C_{\alpha}\left\{\begin{array}{l}
\tau_{0} t_{n+1}^{\alpha-1}, \quad j=0, \\
\tau_{j}\left(t_{n+1}-t_{j}\right)^{\alpha-1}+\tau_{j-1}\left(t_{n+1}-t_{j-1}\right)^{\alpha-1}, \quad j=1,2, \ldots, n+1,
\end{array}\right.
$$

where $C_{\alpha}=\frac{\max \{2, \alpha\}}{\Gamma(\alpha+1)}$.

Lemma 3.5 ([? , Lemma 3.3]) Assume that $\alpha, C_{0}, T>0$ and $b_{j, n}=C_{0} \tau_{j}\left(t_{n}-t_{j}\right)^{\alpha-1}, j=$ $0,1,2, \ldots, n-1$ for $0=t_{0}<t_{1}<\cdots<t_{n}<\cdots<t_{N}=T, n=1,2, \ldots, N$ where $N$ is a positive integer and $\tau_{j}=t_{j+1}-t_{j}$. Let $g_{0}$ be positive and the sequence $\left\{\psi_{k}\right\}$ meet

$$
\left\{\begin{array}{l}
\psi_{0} \leq g_{0} \\
\psi_{n} \leq \sum_{j=1}^{n-1} b_{j, n} \psi_{j}+g_{0}
\end{array}\right.
$$

then

$$
\psi_{n} \leq C g_{0}, \quad n=1,2, \ldots, N
$$

Lemma 3.6 Let $\alpha>0$. We have

(1) $w_{j, n+1}>0, j=0,1,2, \ldots, n$, where $w_{j, n+1}$ are the weights defined in (??).

(2) $\tilde{w}_{j, n+1}>0, j=0,1,2, \ldots, n+1$, where $\tilde{w}_{j, n+1}$ are the weights defined in (??).

Proof. It is obvious that $w_{j, n+1}>0, j=0,1, \ldots, n$. Let us now consider $\tilde{w}_{j, n+1}>0, j=$ $0,1,2, \ldots, n+1$. For $j=0,1,2, \ldots, n$, we have

$$
\tilde{w}_{j, n+1}=\frac{1}{\Gamma(\alpha)} \int_{t_{j}}^{t_{j+1}}\left(t_{n+1}-s\right)^{\alpha-1} \frac{s-t_{j+1}}{t_{j}-t_{j+1}} d s+\frac{1}{\Gamma(\alpha)} \int_{t_{j}}^{t_{j+1}}\left(t_{n+1}-s\right)^{\alpha-1} \frac{s-t_{j}}{t_{j+1}-t_{j}} d s
$$

which is also positive obviously. Further, by (??), we have $\tilde{w}_{n+1, n+1}>0$.

The proof of Lemma?? is complete. 
Proof of Theorem ??. For $n=0,1,2, \ldots, N-1$, we have

$$
\begin{aligned}
\left|y\left(t_{n+1}\right)-y_{n+1}\right|= & \left|\frac{1}{\Gamma(\alpha)} \int_{0}^{t_{n+1}}\left(t_{n+1}-s\right)^{\alpha-1} g(s) d s-\sum_{j=0}^{n} w_{j, n+1} f\left(t_{j}, y_{j}\right)\right| \\
\leq & \frac{1}{\Gamma(\alpha)}\left|\sum_{j=0}^{n} \int_{t_{j}}^{t_{j+1}}\left(t_{n+1}-s\right)^{\alpha-1}\left(g(s)-g\left(t_{j}\right)\right) d s\right| \\
& +\left|\sum_{j=0}^{n} w_{j, n+1}\left(g\left(t_{j}\right)-f\left(t_{j}, y_{j}\right)\right)\right|=I+I I .
\end{aligned}
$$

The term $I$ is estimated by Lemma ??. For $I I$, we have, by Lemma ?? and the Lipschitz condition of $f$,

$$
I I=\left|\sum_{j=0}^{n} w_{j, n+1}\left(g\left(t_{j}\right)-f\left(t_{j}, y_{j}\right)\right)\right| \leq L \sum_{j=0}^{n} w_{j, n+1}\left|y\left(t_{j}\right)-y_{j}\right| .
$$

Thus we obtain

$$
\left|y\left(t_{n+1}\right)-y_{n+1}\right| \leq I+L \sum_{j=0}^{n} w_{j, n+1}\left|y\left(t_{j}\right)-y_{j}\right|
$$

By Lemma ??, we get

$$
\left|y\left(t_{n+1}\right)-y_{n+1}\right| \leq C I
$$

Together these estimates complete the proof of Theorem ??.

\subsection{Trapezoid formula}

In this section we will consider the error estimates of the trapezoid method (??) for solving (??). Assume that ${ }_{0}^{C} D_{t}^{\alpha} y \in C^{2}[0, T]$, Li et al. [?] proved the following theorem

Theorem 3.7 ([? , Theorem 4.2 ]) If ${ }_{0}^{C} D_{t}^{\alpha} y \in C^{2}[0, T]$ and the non-equidistant step size is non-decreasing, then the trapezoid scheme (??) for solving (??) has the following error estimates

$$
\max _{0 \leq j \leq N}\left|y_{j}-y\left(t_{j}\right)\right| \leq C \tau_{\max }^{2}
$$

where $\tau_{\max }$ is defined by (??).

We shall prove the following theorem.

TheOREm 3.8 Let $0<\alpha<2$ and assume that $g:={ }_{0}^{C} D_{t}^{\alpha} y$ satisfies Assumption ??. Let $\tau_{j}, j=0,1,2, \ldots, N-1$ be the non-uniform meshes defined in (??). Assume that $y\left(t_{j}\right)$ and $y_{j}$ are the solutions of (??) and (??), respectively. 
(1) If $0<\alpha<1$, then we have

$$
\max _{0 \leq j \leq N}\left|y\left(t_{j}\right)-y_{j}\right| \leq\left\{\begin{array}{cc}
C N^{-2(\alpha+\sigma)}, & \text { if } 0<2(\alpha+\sigma)<2 \\
C N^{-2(\alpha+\sigma)} \ln (N), & \text { if } 2(\alpha+\sigma)=2 \\
C N^{-2}, & \text { if } 2(\alpha+\sigma)>2
\end{array}\right.
$$

(2) If $1<\alpha<2$, then we have

$$
\max _{0 \leq j \leq N}\left|y\left(t_{j}\right)-y_{j}\right| \leq C N^{-2}
$$

To prove Theorem ??, we need the following lemma.

Lemma 3.9 Let $0<\alpha<2$. Assume that g satisfies Assumption ??.

- If $0<\alpha \leq 1$, then

$$
\left|\frac{1}{\Gamma(\alpha)} \int_{0}^{t_{n+1}}\left(t_{n+1}-s\right)^{\alpha-1} g(s) d s-\sum_{j=0}^{n+1} \tilde{w}_{j, n+1} g\left(t_{j}\right)\right| \leq\left\{\begin{array}{cl}
C N^{-2(\alpha+\sigma)}, & \text { if } 0<2(\alpha+\sigma)<2, \\
C N^{-2(\alpha+\sigma)} \ln (N), & \text { if } 2(\alpha+\sigma)=2, \\
C N^{-2}, & \text { if } 2(\alpha+\sigma)>2 .
\end{array}\right.
$$

- If $1<\alpha<2$, then

$$
\left|\frac{1}{\Gamma(\alpha)} \int_{0}^{t_{n+1}}\left(t_{n+1}-s\right)^{\alpha-1} g(s) d s-\sum_{j=0}^{n+1} \tilde{w}_{j, n+1} g\left(t_{j}\right)\right| \leq C N^{-2}
$$

Proof. Note that, with $n=2,3, \ldots, N-1, N \geq 3$,

$$
\begin{aligned}
& \left|\frac{1}{\Gamma(\alpha)} \int_{0}^{t_{n+1}}\left(t_{n+1}-s\right)^{\alpha-1} g(s) d s-\sum_{j=0}^{n+1} \tilde{w}_{j, n+1} g\left(t_{j}\right)\right| \\
& =\frac{1}{\Gamma(\alpha)}\left|\sum_{j=0}^{n} \int_{t_{j}}^{t_{j+1}}\left(t_{n+1}-s\right)^{\alpha-1}\left(g(s)-\frac{s-t_{j+1}}{t_{j}-t_{j+1}} g\left(t_{j}\right)-\frac{s-t_{j}}{t_{j+1}-t_{j}} g\left(t_{j+1}\right)\right) d s\right| \\
& =\frac{1}{\Gamma(\alpha)}\left|\int_{0}^{t_{1}}\left(t_{n+1}-s\right)^{\alpha-1}\left(g(s)-\frac{s-t_{1}}{-t_{1}} g\left(t_{0}\right)-\frac{s}{t_{1}} g\left(t_{1}\right)\right) d s\right| \\
& \quad+\frac{1}{\Gamma(\alpha)}\left|\sum_{j=1}^{n-1} \int_{t_{j}}^{t_{j+1}}\left(t_{n+1}-s\right)^{\alpha-1}\left(g(s)-\frac{s-t_{j+1}}{t_{j}-t_{j+1}} g\left(t_{j}\right)-\frac{s-t_{j}}{t_{j+1}-t_{j}} g\left(t_{j+1}\right)\right) d s\right| \\
& \quad+\frac{1}{\Gamma(\alpha)}\left|\int_{t_{n}}^{t_{n+1}}\left(t_{n+1}-s\right)^{\alpha-1}\left(g(s)-\frac{s-t_{n+1}}{t_{n}-t_{n+1}} g\left(t_{n}\right)-\frac{s-t_{n}}{t_{n+1}-t_{n}} g\left(t_{n+1}\right)\right) d s\right| \\
& =I_{1}+I_{2}+I_{3} .
\end{aligned}
$$


For $I_{1}$, we have, by Assumption ??,

$$
\begin{aligned}
I_{1} & =\frac{1}{\Gamma(\alpha)}\left|\int_{0}^{t_{1}}\left(t_{n+1}-s\right)^{\alpha-1}\left[\frac{s-t_{1}}{-t_{1}} \int_{0}^{s} g^{\prime}(\tau) d \tau-\frac{s}{t_{1}} \int_{s}^{t_{1}} g^{\prime}(\tau) d \tau\right] d s\right| \\
& \leq C \int_{0}^{t_{1}}\left(t_{n+1}-s\right)^{\alpha-1} s^{\sigma} d s+C \int_{0}^{t_{1}}\left(t_{n+1}-s\right)^{\alpha-1} t_{1}^{\sigma} d s .
\end{aligned}
$$

If $0<\alpha \leq 1$, we have, by (??)

$$
I_{1} \leq C\left(t_{n+1}-t_{1}\right)^{\alpha-1}\left(t_{1}\right)^{\sigma+1} \leq C N^{-2(\alpha+\sigma)} .
$$

If $1<\alpha<2$, then we have, by (??)

$$
I_{1} \leq C t_{n+1}^{\alpha-1} \int_{0}^{t_{1}} s^{\sigma} d s+C t_{n+1}^{\alpha-1} t_{1}^{\sigma+1} \leq C t_{n+1}^{\alpha-1} t_{1}^{\sigma+1} \leq C N^{-2 \sigma-2} \leq C N^{-2} .
$$

For $I_{2}$, we have, with $\xi_{j} \in\left(t_{j}, t_{j+1}\right), j=1,2, \ldots, n-1$ and $n=2,3, \ldots, N-1$,

$$
I_{2} \leq C\left|\sum_{j=1}^{n-1} \int_{t_{j}}^{t_{j+1}}\left(t_{n+1}-s\right)^{\alpha-1} g^{\prime \prime}\left(\xi_{j}\right)\left(s-t_{j}\right)\left(s-t_{j+1}\right) d s\right| .
$$

By Assumption ??, we have, with $n \geq 4$,

$$
\begin{aligned}
I_{2} \leq & C \sum_{j=1}^{n-1}\left(t_{j+1}-t_{j}\right)^{2} t_{j}^{\sigma-2} \int_{t_{j}}^{t_{j+1}}\left(t_{n+1}-s\right)^{\alpha-1} d s \\
\leq & C \sum_{j=1}^{\left\lceil\frac{n-1}{2}\right\rceil-1}\left(t_{j+1}-t_{j}\right)^{2} t_{j}^{\sigma-2} \int_{t_{j}}^{t_{j+1}}\left(t_{n+1}-s\right)^{\alpha-1} d s \\
& +C \sum_{j=\left\lceil\frac{n-1}{2}\right\rceil}^{n-1}\left(t_{j+1}-t_{j}\right)^{2} t_{j}^{\sigma-2} \int_{t_{j}}^{t_{j+1}}\left(t_{n+1}-s\right)^{\alpha-1} d s=I_{21}+I_{22} .
\end{aligned}
$$

Following the similar arguments as in the estimates for $I_{21}$ in lemma ??, we can show, for $0<\alpha \leq 1$,

$$
I_{21} \leq\left\{\begin{array}{cc}
C N^{-2(\alpha+\sigma)}, & \text { if } 0<2(\alpha+\sigma)<2, \\
C N^{-2(\alpha+\sigma)} \ln (N), & \text { if } 2(\alpha+\sigma)=2, \\
C N^{-2}, & \text { if } 2(\alpha+\sigma)>2,
\end{array}\right.
$$

and $I_{21} \leq C N^{-2}$, for $1<\alpha<2$.

Following the similar arguments as in the estimates for $I_{22}$ in lemma ??, we can show, for $0<\alpha \leq 1$,

$$
I_{22} \leq\left\{\begin{array}{cc}
C N^{-2(\alpha+\sigma)}, & \text { if } 0<2(\alpha+\sigma) \leq 2 \\
C N^{-2}, & \text { if } 2(\alpha+\sigma)>2
\end{array}\right.
$$


and $I_{22} \leq C N^{-2}$, for $1<\alpha<2$.

Following the similar arguments as in the estimates for $I_{3}$ in lemma ??, we can show, for $0<\alpha<2$,

$$
I_{3} \leq\left\{\begin{array}{lr}
C N^{-2(\alpha+\sigma)}, & \text { if } 0<2(\alpha+\sigma)<1+\alpha \\
C N^{-(2+\alpha)}, & \text { if } 2(\alpha+\sigma)>1+\alpha .
\end{array}\right.
$$

Together these estimates complete the proof of Lemma ??.

Proof of Theorem ??. For $n=0,1,2, \ldots, N-1$,we have

$$
\begin{aligned}
\left|y\left(t_{n+1}\right)-y_{n+1}\right|= & \left|\frac{1}{\Gamma(\alpha)} \int_{0}^{t_{n+1}}\left(t_{n+1}-s\right)^{\alpha-1} g(s) d s-\sum_{j=0}^{n+1} \tilde{w}_{j, n+1} f\left(t_{j}, y_{j}\right)\right| \\
\leq & \frac{1}{\Gamma(\alpha)}\left|\sum_{j=0}^{n} \int_{t_{j}}^{t_{j+1}}\left(t_{n+1}-s\right)^{\alpha-1}\left(g(s)-\frac{s-t_{j+1}}{t_{j}-t_{j+1}} g\left(t_{j}\right)-\frac{s-t_{j}}{t_{j+1}-t_{j}} g\left(t_{j+1}\right)\right) d s\right| \\
& +\left|\sum_{j=0}^{n+1} \tilde{w}_{j, n+1}\left(g\left(t_{j}\right)-f\left(t_{j}, y_{j}\right)\right)\right| \\
= & I+I I .
\end{aligned}
$$

The term $I$ is estimated by Lemma ??. For $I I$, we have, by Lemma ?? and the Lipschitz condition of $f$,

$$
I I=\left|\sum_{j=0}^{n+1} \tilde{w}_{j, n+1}\left(g\left(t_{j}\right)-f\left(t_{j}, y_{j}\right)\right)\right| \leq L \sum_{j=0}^{n+1} \tilde{w}_{j, n+1}\left|y\left(t_{j}\right)-y_{j}\right| .
$$

Thus we obtain

$$
\left|y\left(t_{n+1}\right)-y_{n+1}\right| \leq I+L \sum_{j=0}^{n+1} \tilde{w}_{j, n+1}\left|y\left(t_{j}\right)-y_{j}\right|
$$

By using the corresponding Gronwall Lemma similar as Lemma ??, see Li et al. [? , Theorem 4.2], we have $\left|y\left(t_{n+1}\right)-y_{n+1}\right| \leq C I$, which completes the proof of Theorem ??.

We remark that for $0<\alpha \leq 1$, we may prove Theorem ?? by using the argument in [? , Lemma 4.3]. However this argument does not work for proving Theorem ?? with $\alpha \in(1,2)$.

\subsection{Predictor-corrector scheme}

In this subsection we will consider the error estimates of the predictor-corrector method (??) for solving (??). Assume that ${ }_{0}^{C} D_{t}^{\alpha} y \in C^{2}[0, T]$, Li et al. [? ] proved the following theorem 
Theorem 3.10 ([? , Theorem 4.3]). If ${ }_{0}^{C} D_{t}^{\alpha} y \in C^{2}[0, T]$ and the non-equidistant step size is non-decreasing, then the predictor-corrector scheme (??) for solving (??) has the following error estimates

$$
\max _{0 \leq j \leq N}\left|y_{j}-y\left(t_{j}\right)\right| \leq C \tau_{\max }^{q}, \quad k=0,1,2, \ldots, N-1,
$$

where $q=\min \{2,1+\alpha\}$ and $\tau_{\max }$ is defined by (??).

We have the following Theorem.

THEOREM 3.11 Let $0<\alpha<2$ and assume that $g:={ }_{0}^{C} D_{t}^{\alpha} y$ satisfies Assumption ??. Let $\tau_{j}, j=0,1,2, \ldots, N-1$ be the non-uniform meshes defined in (??). Assume that $y\left(t_{j}\right)$ and $y_{j}$ are the solutions of (??) and (??), respectively.

(1) If $0<\alpha \leq 1$, then we have

$$
\max _{0 \leq j \leq N}\left|y\left(t_{j}\right)-y_{j}\right| \leq\left\{\begin{array}{cl}
C N^{-2(\alpha+\sigma)}, & \text { if } 2(\alpha+\sigma)<1+\alpha \\
C N^{-2(\alpha+\sigma)} \ln (N), & \text { if } 2(\alpha+\sigma)=1+\alpha \\
C N^{-1-\alpha}, & \text { if } 2(\alpha+\sigma)>1+\alpha
\end{array}\right.
$$

(2) If $1<\alpha<2$, then we have

$$
\max _{0 \leq j \leq N}\left|y\left(t_{j}\right)-y_{j}\right| \leq C N^{-2}
$$

To prove Theorem ??, we need the following lemma.

Lemma 3.12 Let $0<\alpha<2$. Assume that $g(t)$ satisfies Assumption ??.

- If $0<\alpha \leq 1$, then we have

$$
\left|\tilde{w}_{n+1, n+1} \sum_{j=0}^{n} \int_{t_{j}}^{t_{j+1}}\left(t_{n+1}-s\right)^{\alpha-1}\left(g(s)-g\left(t_{j}\right)\right) d s\right| \leq\left\{\begin{array}{cl}
C N^{-2(\alpha+\sigma)}, & \text { if } 2(\alpha+\sigma)<1+\alpha, \\
C N^{-2(\alpha+\sigma)} \ln (N), & \text { if } 2(\alpha+\sigma)=1+\alpha \\
C N^{-1-\alpha}, & \text { if } 2(\alpha+\sigma)>1+\alpha .
\end{array}\right.
$$

- If $1<\alpha<2$, then we have

$$
\left|\tilde{w}_{n+1, n+1} \sum_{j=0}^{n} \int_{t_{j}}^{t_{j+1}}\left(t_{n+1}-s\right)^{\alpha-1}\left(g(s)-g\left(t_{j}\right)\right) d s\right| \leq C N^{-2} .
$$

Proof. The proof is similar to the proof of Lemma ??. We omit the proof here.

Proof of Theorem ??. Following the argument of the proof of [? , Lemma 3.1] and using the estimates in Lemma ??, we may prove this theorem. We omit the proof here.

\section{Numerical examples}

In this section, we will give some numerical examples to illustrate the convergence orders of the numerical methods introduced in the previous sections under the different smooth- 
ness assumptions of ${ }_{0}^{C} D_{t}^{\alpha} y$ in (??). In Example ?? we consider the rectangle formula and in Example ?? we consider the trapezoid formula. In Examples ?? and ?? we consider the predictor-corrector method. We only show the numerical results for $\alpha \in(0,1)$ here. Similarly we may obtain the numerical results for $\alpha \in(1,2)$.

Example 1 Consider, with $0<\alpha<1$,

$$
\begin{aligned}
& { }_{0}^{C} D_{t}^{\alpha} y(t)=f(t, y(t)), \quad t \in(0, T], \\
& y(0)=1,
\end{aligned}
$$

where

$$
f(t, y)=\frac{2}{\Gamma(3-\alpha)} t^{2-\alpha}-\frac{1}{\Gamma(2-\alpha)} t^{1-\alpha}-y^{2}+\left(t^{2}-t+1\right)^{2}
$$

The exact solution is $y(t)=t^{2}-t+1$, and ${ }_{0}^{C} D_{t}^{\alpha} y(t)=\frac{2}{\Gamma(3-\alpha)} t^{2-\alpha}-\frac{1}{\Gamma(2-\alpha)} t^{1-\alpha}$. It is easy to see that ${ }_{0}^{C} D_{t}^{\alpha} y(t) \notin C^{2}[0, T]$ and satisfies Assumption ?? with $\sigma=1-\alpha$.

Let $N \geq 1$ be a positive integer. Let $0=t_{0}<t_{1}<\cdots<t_{N}=T$ be the nonuniform meshes defined in (??). For simplicity, we choose $T=1$. Assume that $y\left(t_{j}\right)$ and $y_{j}, j=0,1,2, \ldots N$ are the solutions of (??) and (??), respectively. By Theorem ??, we have, with $\sigma=1-\alpha$,

$$
\left\|e_{N}\right\|_{\infty}:=\max _{0 \leq j \leq N}\left|y\left(t_{j}\right)-y_{j}\right| \leq C N^{-1}
$$

In Table ??, for the different $\alpha \in(0,1)$, we choose the different $N=20 \times 2^{l}, l=$ $1,2,3,4,5$. We obtain the maximum nodal errors $\left\|e_{N}\right\|_{\infty}$ defined in (??) with respect to the different $N$. We also calculate the experimental order of convergence (EOC) by $\log 2\left(\frac{\left\|e_{N}\right\|_{\infty}}{\left\|e_{2 N}\right\|_{\infty}}\right)$. We observe that the experimental order of convergence is consistent with our theoretical result in (??), i.e, $\log 2\left(\frac{\left\|e_{N}\right\|_{\infty}}{\left\|e_{2 N}\right\|_{\infty}}\right) \approx 1$. In this case, the Caputo fractional derivative ${ }_{0}^{C} D_{t}^{\alpha} y(t)$ behaves as $t^{1-\alpha}, 0<\alpha<1$, by using the rectangle formula with uniform meshes, the experimental order of convergence (EOC) is almost the same as the order obtained by using the rectangle formula with non-uniform meshes.

\begin{tabular}{cccccccc}
\hline Meshes & $\mathrm{N}$ & $\alpha=0.3$ & $\mathrm{EOC}$ & $\alpha=0.6$ & $\mathrm{EOC}$ & $\alpha=0.8$ & $\mathrm{EOC}$ \\
\hline Non-uniform & 40 & $1.51 \mathrm{E}-02$ & & $1.52 \mathrm{E}-02$ & & $1.66 \mathrm{E}-02$ & \\
& 80 & $7.10 \mathrm{E}-03$ & 1.09 & $7.33 \mathrm{E}-03$ & 1.06 & $8.10 \mathrm{E}-03$ & 1.03 \\
& 160 & $3.39 \mathrm{E}-03$ & 1.07 & $3.58 \mathrm{E}-03$ & 1.04 & $4.00 \mathrm{E}-03$ & 1.02 \\
& 320 & $1.63 \mathrm{E}-03$ & 1.06 & $1.76 \mathrm{E}-03$ & 1.02 & $1.99 \mathrm{E}-03$ & 1.01 \\
& 640 & $7.89 \mathrm{E}-04$ & 1.05 & $8.71 \mathrm{E}-04$ & 1.01 & $9.90 \mathrm{E}-04$ & 1.01 \\
Uniform & 40 & $2.44 \mathrm{E}-02$ & & $2.44 \mathrm{E}-02$ & & $2.44 \mathrm{E}-02$ & \\
& 80 & $1.23 \mathrm{E}-02$ & 0.98 & $1.23 \mathrm{E}-02$ & 0.98 & $1.23 \mathrm{E}-02$ & 0.98 \\
& 160 & $6.21 \mathrm{E}-03$ & 0.99 & $6.21 \mathrm{E}-03$ & 0.99 & $6.21 \mathrm{E}-03$ & 0.99 \\
& 320 & $3.12 \mathrm{E}-03$ & 0.99 & $3.12 \mathrm{E}-03$ & 0.99 & $3.12 \mathrm{E}-03$ & 0.99 \\
& 640 & $1.56 \mathrm{E}-03$ & 0.99 & $1.56 \mathrm{E}-03$ & 0.99 & $1.56 \mathrm{E}-03$ & 0.99 \\
\hline
\end{tabular}

Table 1. Maximum nodal errors at $T=1$ for Example ?? using rectangle formula (??) 
EXAmple 2 In this example, we consider the trapezoid formula for (??)-(??). Assume that $y\left(t_{j}\right)$ and $y_{j}, j=0,1,2, \ldots N$ are the solutions of (??) and (??), respectively. By Theorem ??, we have, with $\sigma=1-\alpha$,

$$
\left\|e_{N}\right\|_{\infty}:=\max _{0 \leq j \leq N}\left|y\left(t_{j}\right)-y_{j}\right| \leq C N^{-2} \ln (N) .
$$

In Table ??, for the different $\alpha \in(0,1)$ we choose the different $N=20 \times 2^{l}, l=1,2,3,4,5$. We obtain the maximum nodal errors $\left\|e_{N}\right\|_{\infty}$ defined in (??) with respect to the different $N$. We also calculate the experimental order of convergence (EOC).

\begin{tabular}{cccccccc}
\hline Meshes & $\mathrm{N}$ & $\alpha=0.3$ & $\mathrm{EOC}$ & $\alpha=0.6$ & $\mathrm{EOC}$ & $\alpha=0.8$ & $\mathrm{EOC}$ \\
\hline Non-uniform & 40 & $1.03 \mathrm{E}-04$ & & $1.30 \mathrm{E}-04$ & & $2.18 \mathrm{E}-04$ & \\
& 80 & $2.70 \mathrm{E}-05$ & 1.94 & $3.28 \mathrm{E}-05$ & 1.99 & $5.47 \mathrm{E}-05$ & 2.00 \\
& 160 & $6.97 \mathrm{E}-06$ & 1.95 & $8.24 \mathrm{E}-06$ & 1.99 & $1.37 \mathrm{E}-05$ & 2.00 \\
& 320 & $1.79 \mathrm{E}-06$ & 1.96 & $2.06 \mathrm{E}-06$ & 2.00 & $3.42 \mathrm{E}-06$ & 2.00 \\
& 640 & $4.56 \mathrm{E}-07$ & 1.97 & $5.16 \mathrm{E}-07$ & 2.00 & $8.55 \mathrm{E}-07$ & 2.00 \\
Uniform & 40 & $9.57 \mathrm{E}-04$ & & $4.67 \mathrm{E}-03$ & & $8.30 \mathrm{E}-03$ & \\
& 80 & $4.99 \mathrm{E}-04$ & 0.94 & $2.42 \mathrm{E}-03$ & 0.95 & $4.24 \mathrm{E}-03$ & 0.97 \\
& 160 & $2.62 \mathrm{E}-04$ & 0.93 & $1.25 \mathrm{E}-03$ & 0.96 & $2.15 \mathrm{E}-03$ & 0.98 \\
& 320 & $1.37 \mathrm{E}-04$ & 0.93 & $6.35 \mathrm{E}-04$ & 0.97 & $1.08 \mathrm{E}-03$ & 0.99 \\
& 640 & $7.13 \mathrm{E}-05$ & 0.94 & $3.22 \mathrm{E}-04$ & 0.98 & $5.44 \mathrm{E}-04$ & 0.99 \\
\hline
\end{tabular}

Table 2. Maximum nodal errors at $T=1$ for Example ?? using trapezoid formula (??)

We see that the experimental order of convergence (EOC) of the trapezoid method (??) with non-uniform meshes is almost $O\left(N^{-2}\right)$ as we expected. However the experimental order of convergence (EOC) of the trapezoid method (??) with uniform meshes is only about $O\left(N^{-1}\right)$

Example 3 Consider, with $0<\alpha<1,0<\beta<1$ and $\alpha<\beta$,

$$
\begin{aligned}
& { }_{0}^{C} D_{t}^{\alpha} y(t)=\frac{\Gamma(1+\beta)}{\Gamma(1+\beta-\alpha)} t^{\beta-\alpha}+t^{2 \beta}-y^{2}, \quad t \in(0, T], \\
& y(0)=y_{0},
\end{aligned}
$$

where $y_{0}=0$, and the exact solution is $y(t)=t^{\beta}$, and ${ }_{0}^{C} D_{t}^{\alpha} y(t)=\frac{\Gamma(1+\beta)}{\Gamma(1+\beta-\alpha)} t^{\beta-\alpha}$, which implies that the regularity of ${ }_{0}^{C} D_{t}^{\alpha} y(t)$ behaves as $t^{\beta-\alpha}$. Thus we see that ${ }_{0}^{C} D_{t}^{\alpha} y(t)$ satisfies the Assumption 1 with $\sigma=\beta-\alpha$.

We shall use the same notations as in Example ??. We have, by Theorem ?? with $\sigma=\beta-\alpha$,

$$
\left\|e_{N}\right\|:=\max _{0 \leq j \leq N}\left|y\left(t_{j}\right)-y_{j}\right| \leq\left\{\begin{array}{cl}
C N^{-2 \beta}, & \text { if } 2 \beta<1+\alpha, \\
C N^{-2 \beta} \ln (N), & \text { if } 2 \beta=1+\alpha, \\
C N^{-(1+\alpha)}, & \text { if } 2 \beta>1+\alpha .
\end{array}\right.
$$

In Table ??, we choose $\beta=0.9$ and we obtain the experimental orders of convergence (EOC) and the maximum nodal errors with respect to the different $N$. In Table ??, we have $2 \beta \geq 1+\alpha$, the experimental order of convergence (EOC) of (??) with non-uniform meshes is almost $1+\alpha$ as we expected. 


\begin{tabular}{cccccccc}
\hline Meshes & $\mathrm{N}$ & $\alpha=0.3$ & $\mathrm{EOC}$ & $\alpha=0.6$ & $\mathrm{EOC}$ & $\alpha=0.8$ & $\mathrm{EOC}$ \\
\hline Non-uniform & 40 & $1.74 \mathrm{E}-02$ & & $1.53 \mathrm{E}-03$ & & $5.19 \mathrm{E}-04$ & \\
& 80 & $5.36 \mathrm{E}-03$ & 1.70 & $4.57 \mathrm{E}-04$ & 1.75 & $1.49 \mathrm{E}-04$ & 1.80 \\
& 160 & $1.73 \mathrm{E}-03$ & 1.63 & $1.41 \mathrm{E}-04$ & 1.70 & $4.28 \mathrm{E}-05$ & 1.80 \\
& 320 & $5.90 \mathrm{E}-04$ & 1.55 & $4.46 \mathrm{E}-05$ & 1.66 & $1.23 \mathrm{E}-05$ & 1.80 \\
& 640 & $2.10 \mathrm{E}-04$ & 1.49 & $1.43 \mathrm{E}-05$ & 1.64 & $3.53 \mathrm{E}-06$ & 1.80 \\
Uniform & 40 & $7.39 \mathrm{E}-02$ & & $8.95 \mathrm{E}-03$ & & $1.43 \mathrm{E}-02$ & \\
& 80 & $2.37 \mathrm{E}-02$ & 0.85 & $4.83 \mathrm{E}-03$ & 0.89 & $7.68 \mathrm{E}-03$ & 0.90 \\
& 160 & $7.08 \mathrm{E}-03$ & 0.88 & $2.59 \mathrm{E}-03$ & 0.90 & $4.12 \mathrm{E}-03$ & 0.90 \\
& 320 & $2.24 \mathrm{E}-03$ & 0.89 & $1.39 \mathrm{E}-03$ & 0.90 & $2.21 \mathrm{E}-03$ & 0.90 \\
& 640 & $7.62 \mathrm{E}-04$ & 0.90 & $7.46 \mathrm{E}-04$ & 0.90 & $1.18 \mathrm{E}-03$ & 0.90 \\
\hline
\end{tabular}

Table 3. Maximum nodal errors at $T=1$ for Example ?? using predictor-corrector scheme (??) with $\beta=0.9$

In Table ??, we choose $\beta=0.4$ and we obtain the experimental orders of convergence $(E O C)$ and the maximum nodal errors with respect to the different $N$. In Table ??, we have $2 \beta<1+\alpha$, the experimental order of convergence (EOC) of (??) with nonuniform meshes is indeed almost $2 \beta=0.8$ as we expected for $\alpha=0.2,0.3$. For $\alpha=0.1$, the experimental order of convergence (EOC) of (??) with non-uniform meshes is less than $2 \beta=0.8$. If we choose $\alpha>0.5$ in this case, we could not get any numerical results, the solutions blow up.

\begin{tabular}{cccccccc}
\hline Meshes & $\mathrm{N}$ & $\alpha=0.3$ & $\mathrm{EOC}$ & $\alpha=0.2$ & $\mathrm{EOC}$ & $\alpha=0.1$ & $\mathrm{EOC}$ \\
\hline Non-uniform & 40 & $6.06 \mathrm{E}-03$ & & $3.21 \mathrm{E}-02$ & & $4.63 \mathrm{E}-01$ & \\
& 80 & $2.15 \mathrm{E}-03$ & 1.49 & $7.68 \mathrm{E}-03$ & 2.06 & $4.09 \mathrm{E}-01$ & 0.18 \\
& 160 & $1.08 \mathrm{E}-03$ & 0.99 & $2.08 \mathrm{E}-03$ & 1.88 & $3.48 \mathrm{E}-01$ & 0.23 \\
& 320 & $5.40 \mathrm{E}-04$ & 0.99 & $1.21 \mathrm{E}-03$ & 0.78 & $2.76 \mathrm{E}-01$ & 0.33 \\
\multirow{5}{*}{ Uniform } & 640 & $2.70 \mathrm{E}-04$ & 0.99 & $6.97 \mathrm{E}-04$ & 0.79 & $1.76 \mathrm{E}-01$ & 0.65 \\
& 40 & $2.02 \mathrm{E}-02$ & & $1.14 \mathrm{E}-02$ & & $4.02 \mathrm{E}-01$ & \\
& 80 & $1.64 \mathrm{E}-02$ & 0.30 & $9.96 \mathrm{E}-03$ & 0.20 & $3.41 \mathrm{E}-01$ & 0.23 \\
& 160 & $1.25 \mathrm{E}-02$ & 0.40 & $1.05 \mathrm{E}-02$ & -0.07 & $2.70 \mathrm{E}-01$ & 0.34 \\
& 320 & $9.18 \mathrm{E}-03$ & 0.44 & $9.40 \mathrm{E}-03$ & 0.16 & $1.77 \mathrm{E}-01$ & 0.61 \\
& 640 & $6.63 \mathrm{E}-03$ & 0.47 & $7.86 \mathrm{E}-03$ & 0.26 & $1.14 \mathrm{E}-02$ & 0.96 \\
\hline
\end{tabular}

Table 4. Maximum nodal errors at $T=1$ for Example ?? using predictor-corrector scheme (??) with $\beta=0.4$

ExAmple 4 Consider, with $0<\alpha<1$,

$$
\begin{aligned}
& { }_{0}^{C} D_{t}^{\alpha} y(t)+y(t)=0, \quad t \in(0, T], \\
& y(0)=y_{0},
\end{aligned}
$$

where $y_{0}=1$. The exact solution is $y(t)=E_{\alpha, 1}\left(-t^{\alpha}\right)$, and ${ }_{0}^{C} D_{t}^{\alpha} y(t)=-E_{\alpha, 1}\left(-t^{\alpha}\right)$, where $E_{\alpha, \gamma}(z)$ is the Mittag-Leffler function defined by

$$
E_{\alpha, \gamma}(z)=\sum_{k=0}^{\infty} \frac{z^{k}}{\Gamma(\alpha k+\gamma)}, \quad \alpha, \gamma>0
$$


Hence we have

$$
{ }_{0}^{C} D_{t}^{\alpha} y(t)=-1-\frac{\left(-t^{\alpha}\right)}{\Gamma(\alpha+1)}-\frac{\left(-t^{\alpha}\right)^{2}}{\Gamma(2 \alpha+1)}-\ldots
$$

which implies that the regularity of ${ }_{0}^{C} D_{t}^{\alpha} y(t)$ behaves as $c+c t^{\alpha}, 0<\alpha<1$. By Theorem ?? with $\sigma=\alpha$, we have

$$
\left\|e_{N}\right\|_{\infty}:=\max _{0 \leq j \leq N}\left|y\left(t_{j}\right)-y_{j}\right| \leq\left\{\begin{array}{cc}
C N^{-4 \alpha}, & \text { if } \alpha<1 / 3 \\
C N^{-4 \alpha} \ln (N), & \text { if } \alpha=1 / 3 \\
C N^{-(1+\alpha)}, & \text { if } \alpha>1 / 3
\end{array}\right.
$$

In Table ?? we obtain the experimental orders of convergence (EOC) and the maximum nodal errors with respect to the different $N$. When $\alpha=0.3$ which is less than $1 / 3$, the convergence order should be $O\left(N^{-4 \alpha}\right)=O\left(N^{-1.2}\right)$. When $\alpha=0.6,0.8$ which are greater than $1 / 3$, the convergence orders should be $1+\alpha$. We see that the numerical results are perfectly consistent with the theoretical results for $\alpha=0.6,0.8$. For $\alpha=$ 0.3 , the experimental orders of convergence $(E O C)$ is slightly lower than the theoretical convergence order $O\left(N^{-1.2}\right)$.

\begin{tabular}{cccccccc}
\hline Meshes & $\mathrm{N}$ & $\alpha=0.3$ & $\mathrm{EOC}$ & $\alpha=0.6$ & $\mathrm{EOC}$ & $\alpha=0.8$ & $\mathrm{EOC}$ \\
\hline Non-uniform & 40 & $9.50 \mathrm{E}-04$ & & $4.26 \mathrm{E}-04$ & & $2.53 \mathrm{E}-04$ & \\
& 80 & $4.87 \mathrm{E}-04$ & 0.96 & $1.35 \mathrm{E}-04$ & 1.66 & $7.23 \mathrm{E}-05$ & 1.81 \\
& 160 & $2.64 \mathrm{E}-04$ & 0.88 & $4.37 \mathrm{E}-05$ & 1.63 & $2.08 \mathrm{E}-05$ & 1.80 \\
& 320 & $1.31 \mathrm{E}-04$ & 1.02 & $1.42 \mathrm{E}-05$ & 1.62 & $6.01 \mathrm{E}-06$ & 1.79 \\
Uniform & 640 & $6.14 \mathrm{E}-04$ & 1.09 & $4.66 \mathrm{E}-06$ & 1.61 & $1.74 \mathrm{E}-06$ & 1.79 \\
& 40 & $1.11 \mathrm{E}-02$ & & $7.57 \mathrm{E}-04$ & & $1.14 \mathrm{E}-04$ & \\
& 80 & $4.47 \mathrm{E}-03$ & 1.32 & $4.34 \mathrm{E}-04$ & 0.80 & $4.43 \mathrm{E}-05$ & 1.37 \\
& 160 & $1.24 \mathrm{E}-03$ & 1.84 & $2.19 \mathrm{E}-04$ & 0.98 & $1.59 \mathrm{E}-05$ & 1.48 \\
& 320 & $5.54 \mathrm{E}-04$ & 1.17 & $1.05 \mathrm{E}-04$ & 1.07 & $5.47 \mathrm{E}-06$ & 1.54 \\
& 640 & $6.48 \mathrm{E}-04$ & -0.22 & $4.83 \mathrm{E}-05$ & 1.12 & $1.85 \mathrm{E}-06$ & 1.56 \\
\hline
\end{tabular}

Table 5. Maximum nodal errors at $T=1$ for Example ?? using predictor-corrector scheme (??)

\subsection{Conclusion}

In this paper, we consider the error estimates of three kinds of fractional numerical methods for solving nonlinear fractional differential equations with non-uniform meshes. Under the assumption that the fractional derivative of the solution of the fractional differential equation has lower smoothness, we obtain the error bounds which depend on the fractional order $\alpha$ and the smoothness of the Caputo fractional derivative of the solution of the fractional differential equation. We may extend this idea to consider other numerical methods for solving fractional ordinary or partial differential equations with non-uniform meshes. 


\section{Acknowledgment}

The work of the first author was carried out during her stay at the University of Chester, which is supported financially by Shanxi province government, P. R. China. She thanks the Department of Mathematics, University of Chester for its warm hospitality and providing a very good working condition for her during her stay in Chester.

\section{References}

[1] J. Cao and $\mathrm{C} . \mathrm{Xu}$, A high order schema for the numerical solution of the fractional ordinary differential equations, J. Comput. Phys., 238(2013), 154-168.

[2] W. H. Deng, Short memory principle and a predictor-corrector approach for fractional differential equations, J. Comput. Appl. Math., 206(2007), 1768-1777.

[3] K. Diethelm, The Analysis of Fractional Differential Equations, An Application-Oriented Exposition Using Differential Operators of Caputo Type, Lecture Notes in Mathematics, Springer, (2010).

[4] K. Diethelm, N.J. Ford, Analysis of fractional differential equations, J. Math. Anal. Appl. 265 (2) (2002), $229-248$.

[5] K. Diethelm, N.J. Ford and A.D. Freed, Detailed error analysis for a fractional Adams method, Numer. Algor., 36(2004), 31-52.

[6] K. Diethelm, N. J. Ford and A.D. Freed, A predictor-corrector approach for the numerical solution of fractional differential equations, Nonlinear Dynamics, 29(2002), 3-22.

[7] A. A. Kilbas, H. M. Srivastava, J. J. Trujillo, Theory and Applications of Fractional Differential Equations, Elsevier, 2006.

[8] M. Kolk, A. Pedas and E. Tamme, Modified spline collocation for linear fractional differential equations, J. Comput. Appl. Math., 283(2015), 28-40.

[9] C. Li, Q. Yi and A. Chen, Finite difference methods with non-uniform meshes for nonlinear fractional differential equations, J. Comput. Phys., 316(2016), 614-631.

[10] Z. Li, Y. Yan and N. J. Ford, Error estimates of a high order numerical method for solving linear fractional differential equation, Applied Numerical Mathematics, 114(2017), 201-220.

[11] C. Li and C. Tao, On the fractional Adams method, Comput. Math. Appl. 58(2009), 1573-1588.

[12] C. Li and F. Zeng, The finite difference methods for fractional ordinary differential equations, Numer. Funct. Anal. Optim., 34(2013), 149-179.

[13] C. Lubich, Runge-Kutta theory for Volterra and Abel integral equations of the second kind, Math. Comp., 41(1983), 87-102.

[14] K. Oldham and J. Spanier, The Fractional Calculus, Academic Press, San Diego, 1974.

[15] K. Pal, F. Liu and Y. Yan, Numerical solutions for fractional differential equations by extrapolation, Lecture Notes in Computer Science, Springer series, 9045(2015), 299-306.

[16] A. Pedas and E. Tamme, Numerical solution of nonlinear fractional differential equations by spline collocation methods, J. Comput. Appl. Math., 255(2014), 216-230.

[17] J. Quintana-Murillo and S. B. Yuste, A finite difference method with non-uniform timesteps for fractional diffusion and diffusion-wave equations, The European Physical Journal Special Topics, 222(2013), 1987-1998.

[18] I. Podlubny, Fractional Differential Equations, Mathematics in Science and Engineering, Vol. 198, Academic Press, 1999.

[19] M. Stynes, Too much regularity may force too much uniqueness, Fractional Calculus and Applied Analysis, 19(2016), 1554-1562.

[20] M. Stynes, E. O'riordan and J. L. Gracia, Error analysis of a finite difference method on graded meshes for a time-fractional diffusion equation, SIAM J. Numer. Anal., 55(2017), 1057-1079.

[21] Y. Yan, K. Pal and N. J. Ford, Higher order numerical methods for solving fractional differential equations, BIT Numer. Math., 54(2014), 555-584.

[22] S. B. Yuste and J. Quintana-Murillo, Fast, accurate and robust adaptive finite difference methods for fractional diffusion equations, Numer. Algor., 71(2016), 207-228.

[23] Y. Zhang, Z. Sun and H. Liao, Finite difference methods for the time fractional diffusion equation on non-uniform meshes, J. Comput. Phys., 265(2014), 195-210.

[24] L. Zhao and W. H. Deng, Jacobian-predictor-corrector approach for fractional ordinary differential equations, Adv. Comput. Math., 40(2014), 137-165. 